\title{
A Review of Ten-Years Studies on the Microbial Carbon Pump
}

\author{
NIANZHI JIAO ${ }^{1}$ \\ ${ }^{1}$ State Key Laboratory of Marine Environmental Science, \\ Xiamen University, Xiamen 361005, China \\ (jiao@xmu.edu.cn)
}

Microbial Carbon Pump (MCP) was proposed in 2010 as a conceptual framework to address the mechanisms underpinning the formation of refractory dissolved organic carbon (RDOC) in the ocean which is closely related to climate changes. Building on conventional concepts of the biological pump (BP) and microbial loop (ML), MCP is concerned with both microbial uptakes and outputs of DOC compounds, emphasizing the microbial transformation of organic carbon from labile to recalcitrant states. The MCP products can be classified into two categories, environmental context-dependent RDOC $_{t}$ and concentration-constrained RDOC $\left(\right.$ RDOC $\left._{c}\right)$. During the past ten years, the MCP concept has aroused interests from multiple research disciplines and been scrutinized in the scientific community. MCP has been studied in different regions covering coastal and oceanic waters and at different spatial and temperate scales, which substantially promoted our understanding of the microbial roles in global ocean carbon cycle. In particular, under future global warming scenarios, partitioning of primary production into DOC could be enhanced, and thus the MCP could play an even more important role in ocean carbon sequestration. In this review, I will illustrate the differences and connections between MCP and BP/ML based on practical studies, and show the research trends of MCP, BP and ML as seen from literature data analysis. I will then summarize major breakthroughs in MCP studies over the past decade, including a few milestones of the process: MCP concept proposal (2010); refined classification of the MCP products; RDOCt and RDOCc (2014); debates on who's the majority of the two (2015); experimental test of the efficiency of MCP transformation from labile DOC to RDOC through megacosm long term incubation (2018); and inclusion of MCP in the IPCC Special Report on Ocean and Cryosphere (2019). As a newly proposed theoretical framework, MCP is open to more in-depth studies, such as precise composition of the RDOC components and the MCP processes producing them. Meanwhile MCP is of great potential for carbon sequestration practices, and worth of study on how to enhance the overall effects of MCP and BP for carbon sink eco-engineering in the coastal and oceanic waters. 